\title{
Shared brain activity for aesthetic and moral judgments: implications for the Beauty-is-Good stereotype
}

\author{
Jorge Faber*, Patrícia Medeiros Berto**
}

The Beauty-is-Good stereotype refers to the assumption that attractive people possess sociably desirable personalities and higher moral standards. The existence of this bias suggests that the neural mechanisms for judging facial attractiveness and moral goodness overlap, i.e., they are circumscribed to the same brain regions. The hypothesis of this overlap was investigated by Tsukiura and Cabeza ${ }^{1}$ and published in the March 2010 issue of the Journal of Social Cognitive and Affective Neuroscience.

The research participants were scanned with functional magnetic resonance imaging while they made attractiveness judgments about faces and goodness judgments about hypothetical actions. Activity in the medial orbitofrontal cortex increased as a function of both attractiveness and goodness ratings, whereas activity in the insular cortex decreased with both attractiveness and goodness ratings. These activations support the idea of similar contributions of each region to both judgments. The orbitofrontal and insular cortices were negatively correlated with each other, suggesting an opposing relationship between these regions during attractiveness and goodness judgments.

These findings have implications for understanding the neural mechanisms of the Beautyis-Good stereotype. People judged to be physically attractive often have their personality also judged positively, be it as a person of good conduct, virtuous or even honest. One is capable of, at first sight, considering another human being attractive or unattractive while at the same time assigning values to that person. The study suggests a possible explanation for this fact since the same neural mechanisms are activated or deactivated during these types of assessments. So, perhaps now, we can explain why, when a person is seen as beautiful, they are likewise seen as good. In other words, how beauty becomes goodness.

\footnotetext{
* Editor-in-Chief of the Dental Press Journal of Orthodontics. PhD in Biology - Morphology, Electronic Microscopy Laboratory, University of

Brasília (UnB). MSc in Orthodontics and Dentofacial Orthopedics, UFRJ.

$\star \star$ Specialist in Orthodontics, Federal University of Goiás (UFG). Reviewer of the Dental Press Journal of Orthodontics.
} 


\section{Facial expressions and how the brain decodes them}

It is a fact that sociable living beings are able to perceive the social cues of their peers. The same applies to humans. In primates, the face has evolved to convey emotional states, while the brain has simultaneously evolved to decode the signals in the facial expressions of others. The study by Schyns, Petro and Smith ${ }^{2}$ reviewed and integrated the evidence supporting this hypothesis.

With the aid of computer programs they co-examined facial expressions as signals that transmit information and the brain as a receiver and decoder of these signals. The authors found that facial expressions were a set of subtly correlated signals, i.e., only slightly resembling one another. For example, the eyes can share similar expressions of anger and happiness. Data from EEG's showed that the brain uses spatial frequency information that reaches the retina to identify the expressions by breaking up their correlations. Within 140 to 200 milliseconds after stimulus onset-regardless of the expression or the brain hemisphere side-an information processing mechanism is triggered locally to take motor control of the eyes. The eyes then perform a wide zoom to process the entire face and finally a close-up zoom on specific spots for diagnostic purposes (e.g., eyes open in "fear"; mouth opens in "happiness").

A categorizing model showed that in 200 milliseconds the left and right hemispheres process enough information to predict the behavioral category of the face being analyzed. This investigation contributes to the understanding of how facial information is quickly processed in the brain to identify emotions.

Research of this nature, which enhance our understanding of how beauty is recognized, will probably be useful in establishing treatment strategies that involve aesthetic reconstruction of the face or its subcomponents, such as the smile.

\section{Motivation and enthusiasm over orthognathic surgery results influence treatment satisfaction}

Patients' motivation to undergo orthognathic surgery can affect their satisfaction with treatment outcome. Meade and Inglehart ${ }^{3}$ investigated this relationship and published their findings in the American Journal of Orthodontics and Dentofacial Orthopedics. The goal was to determine whether the expectations of patients and their parents regarding their possible future appearance were correlated with the patients' treatment satisfaction.

A retrospective study was performed and questionnaires were presented to 115 patients 
(aged 13-21 years at surgery) and 117 parents (response rates of $41 \%$ and $42 \%$ respectively), with responses from 95 parent-patient pairs. The patients' motivation was evaluated before surgery by determining how excited they were when they envisioned themselves after surgery and how focused they were on the results. Parents completed parallel questionnaires on their children's motivation. Patient satisfaction was determined by means of a postsurgical satisfaction questionnaire. The data collected indicated that the more excited the patients were before surgery, the more satisfied they were with the results. Likewise, the more these patients focused on functional and aesthetic changes, the more satisfied they were with the results. The assessments made by the parents regarding the motivation of their children before surgery were consistent with the children's reports and correlated with patient satisfaction after the surgery. Thus, young patients' self-motivation towards orthognathic surgery is strongly correlated with their treatment satisfaction.

The findings of this study have clinical implications for maxillofacial surgeons and orthodontists. Attention to technical excellence and the use of advanced technologies are currently the day-to-day concerns of most practitioners. They are indeed essential for ensuring a successful surgery. However, patient satisfaction should be added to the technical requirements of a surgery-it is possible the coexistence of a surgery that meets the technical criteria and a patient dissatisfied with its results, and this would be a scenario of failure. What the article suggests is the need to evaluate and encourage patients about the surgery results from the very first appointment in the pre-operative phase. The more motivated and focused are the patients, the more likely they are to experience ultimate success. Such evidence can, no doubt, be readily applied in our daily professional practice.

\section{REFERENCES}

1. Tsukiura T, Cabeza R. Shared brain activity for aesthetic and moral judgments: implications for the Beauty-is-Good stereotype. Soc Cogn Affect Neurosci. 2010 Mar 15. [Epub ahead of print].

2. Schyns PG, Petro LS, Smith ML. Transmission of facial expressions of emotion co-evolved with their efficient decoding in the brain: behavioral and brain evidence. PLoS One. 2009 May 20;4(5):e5625.

3. Meade EA, Inglehart MR. Young patients' treatment motivation and satisfaction with orthognathic surgery outcomes: the role of possible selves. Am J Orthod Dentofacial Orthop. 2010 Jan;137(1):26-34.

Contact address

Jorge Faber

Brasília Shopping Torre Sul sala 408

CEP: 70.715-900 - Brasília/DF

E-mail: faber@dentalpress.com.br 